\title{
Quantum dot with spin-orbit interaction in noncommutative phase space and analog Landau levels
}

\author{
B.K.Pal $\stackrel{*}{,}$ B. Roy ${ }^{\dagger}$ B. Basu $\ddagger$ \\ Physics \& Applied Mathematics Unit \\ Indian Statistical Institute \\ Calcutta 700108 \\ India
}

February 6, 2020

\begin{abstract}
We have studied a quantum dot with Rashba spin orbit interaction on a plane where the position and momentum coordinates are considered to be noncommutative. The energy spectrum of the system is found to be equivalent to that of a quantum dot with Rashba spin-orbit interaction in a magnetic field under certain conditions.
\end{abstract}

\section{Introduction}

In recent years considerable attention has been paid to the investigation of different physical problems in noncommutative spaces [1]. The reasons for the emergence of this interest were the predictions of String theory [2] in the low-energy limit which, along with the Brane-world scenario [3], led to the fact the space-time could be noncommutative. Later intensive investigation of the field theory on noncommutative spaces was prompted by Mtheory [4] and the matrix formulation of the quantum Hall effect [5]. It has been shown that noncommutative spaces may arise as the gravitation quantum efeect and and may

\footnotetext{
*E-mail: barunpal1985@rediffmail.com

$\dagger$ E-mail : barnana@isical.ac.in

${ }^{\ddagger}$ E-mail: banasri@isical.ac.in Fax:91+(033)2577-3026
} 
serve as a possible way for the regularization of quantum field theories [6]. By considering the low-energy limit of one-particle sector of field theory on noncommutative space one arrives at what is called noncommutative quantum mechanics. Although the noncommutative scale is expected to be pretty small, perhaps below the planck scale [7], people are looking for phenomenological consequences of the noncommutative geometry in low energy quantum mechanical regime. Many interesting quantum mechanical problems on noncommutative spaces have been investigated and the effect of noncommutativity on the observables has been analyzed [8].

On the other hand, in recent years, the physics concerning the spin of electrons has become a widely studied subject in the context of semiconductor physics. The fundamental physics involved and the possibility of future technological applications motivate this research . In particular, the role of the spin in low dimensional semiconductor structures like quantum dots, is especially attractive because of the ability to control the relevant parameters of the nanostructures available at present. Thus it is possible to have knowledge of the interactions governing the spin dynamics within these structures. Among the interactions concerning the spin, a relevant intrinsic interaction in non-magnetic semiconductors is the spin-orbit coupling, which has been extensively studied in the context of quantum dots [9]. This interaction stems from the relativistic correction to the electron motion. The electric fields present in the semiconductor are felt by the electrons in their intrinsic reference frame as a spin-dependent magnetic field whose intensity depends on the momentum of the particle. Depending on the origin of the electric field there are two types of spin-orbit coupling: the lack of inversion symmetry in a bulk semiconductor gives rise to the so-called Dresselhaus term [10], whereas the asymmetry of the potential confining the two-dimensional electron gas of a heterostructure is responsible for BychkovRashba interaction [11]. The other spin-dependent interaction is the Zeeman interaction arising from the direct coupling of the intrinsic magnetic moment associated with the spin with an applied magnetic field. In this regard, the analytical solutions for quantum dots with Rashba spin-orbit interaction for diferent confinement potential is also studied $[12,13,14]$.

In this note, we shall consider a quantum dot with Rashba spin-orbit coupling in a space where both position and momentum coordinates are noncommutative. The reason for taking the momentum noncommutativity comes from the fact that in quantum mechanics, the generalized momentum components are noncommutative. Moreover, it has been shown recently [15] that in order to keep the Bose-Einstein statistics for identical particles intact at the noncommutative level, one should consider both the space-space and momentum-momentum noncommutativity.

This note is organized as follows: In Section 2 we consider a quantum dot with harmonic confinement and Rashba spin-orbit interaction in a two dimensional space where both the position and momentum are noncommutative. The energy spectrum of the system is found and its degeneracy is discussed for specific noncommutative parameter ranges. In Section 3 the model of Section 2 is compared with a system consisting of a quantum dot with Rashba 
spin-orbit interaction in a magnetic field and a relation between the noncommutative parameters $\theta$ and $\bar{\theta}$ with the magnetic field and Rashba spin-orbit interaction coupling strength $\lambda_{R}$ is established. Finally, the concluding remarks are given in Section 4.

Thorughout our calculations we have used the natural unit system $(\hbar=c=1)$ and we have retained the terms upto the order of $\lambda_{R}^{2}$.

\section{Quantum dot with spin-orbit interaction in non- commutative space}

In the presence of Rashba spin orbit interaction, the Hamiltonian of a single electron state of a quantum dot with harmonic confinement is given by,

$$
H=-\frac{1}{2 m}\left(\frac{\partial^{2}}{\partial x^{2}}+\frac{\partial^{2}}{\partial y^{2}}\right)+\frac{m}{2} \omega_{0}^{2}\left(x^{2}+y^{2}\right)+i \lambda_{R}\left(\sigma_{x} \frac{\partial}{\partial y}-\sigma_{y} \frac{\partial}{\partial x}\right)
$$

where $\lambda_{R}$ is the Rashba spin-orbit coupling parameter.

In non-commutative spacetime the ordinary product is replaced by a star product of the form

$$
\psi(x) \star \phi(x)=\left.\exp \left\{i \theta_{\mu \nu} \partial_{x}^{\mu} \partial_{y}^{\nu}\right\} \psi(x) \phi(x)\right|_{x=y}
$$

where $\theta_{\mu \nu}$ is an antisymmetric constant matrix. As $\theta_{0 i} \neq 0$ leads to a non-unitary theory [16], only the elements $\theta_{i j}, i, j=1,2,3$ are allowed to be nonvanishing. In order not to spoil the isotropy os space it is mandatory to choose $\theta_{i j}$ proportional to the constant antisymmetric matrix $\epsilon, \theta_{i j}=\theta \varepsilon_{i j}$, where $\theta$, the noncommutativity parameter, is a constant with dimension of $(\text { length })^{2}$ and

$$
\epsilon=\left(\begin{array}{ccc}
0 & -1 & 1 \\
1 & 0 & -1 \\
-1 & 1 & 0
\end{array}\right)
$$

This leads to the Moyal comutation relations between the spatial and momentum coordinates as

$$
\begin{aligned}
{\left[\bar{x}_{i}, \bar{x}_{j}\right] } & =2 i \varepsilon_{i j} \theta \\
{\left[\bar{p}_{i}, \bar{p}_{j}\right] } & =2 i \varepsilon_{i j} \bar{\theta} \\
{\left[\bar{x}_{i}, \bar{p}_{j}\right] } & =i \delta_{i j}[1+\theta \bar{\theta}]
\end{aligned}
$$

Here $\theta$ is the non commutative parameter for the co-ordinate space and $\bar{\theta}$ is the non commutative parameter for the momentum space. The non-commutative Hamiltonian is then given by,

$$
\bar{H}=\frac{1}{2 m} \overline{\mathbf{p}}^{2}+\frac{1}{2} m \omega_{0}^{2} \overline{\mathbf{r}}^{2}+\lambda_{R}\left(\sigma_{x} \overline{p_{y}}-\sigma_{y} \overline{p_{x}}\right)
$$


Now the star product (2) can be replaced by a shift called Bopp's shift [17] so that the non-commutative operators and commutative operators are related via the following relation:

$$
\begin{array}{cc}
\bar{x}=x-\theta p_{y} & , \quad \bar{y}=y+\theta p_{x} \\
\overline{p_{x}}=p_{x}+\bar{\theta} y & , \quad \overline{p_{y}}=p_{y}-\bar{\theta} x .
\end{array}
$$

Hence, the above non-commutative Hamiltonian in the commutative space can be written as:

$H_{N C}=A\left(p_{x}^{2}+p_{y}^{2}\right)+B\left(x^{2}+y^{2}\right)+C\left(y p_{x}-x p_{y}\right)+\lambda_{R}\left[\left(p_{y} \sigma_{x}-\sigma_{y} p_{x}\right)-\bar{\theta}\left(x \sigma_{x}+y \sigma_{y}\right)\right]=H_{0}+H_{\text {spin }}$

where,

$$
\begin{aligned}
& A=\left(\frac{1}{2 m}+\frac{m \omega_{0}^{2} \theta^{2}}{2}\right), \\
& B=\left(\frac{\bar{\theta}^{2}}{2 m}+\frac{m \omega_{0}^{2}}{2}\right), \\
& C=\left(\frac{\bar{\theta}}{m}+\theta m \omega_{0}^{2}\right),
\end{aligned}
$$

By means of an unitary transformation $[18,19]$ the above Hamiltonian can be diagonalised in spin space upto second order in the spin-orbit parameter $\lambda_{R}$. In order to neglect higher order terms introduced by the unitary transformation it is required that the energy scale correponding to the spin orbit interaction must be much smaller than that corresponding to the spin independent Hamiltonian $H_{0}>>H_{\text {spin }}$. The Hamiltonian (6) in the transformed reference frame is given by

$$
H^{\prime}=U^{\dagger} H_{N C} U
$$

where,

$$
\begin{aligned}
U & =e^{-i \beta \hat{O}} \\
\hat{O} & =\left(p_{x}-\frac{y}{\theta}\right) \sigma_{x}+\left(p_{y}-\frac{x}{\theta}\right) \sigma_{y} \\
\beta & =\frac{\lambda_{R}}{\frac{\theta}{m}-\frac{1}{m \theta}}
\end{aligned}
$$

We expand the transformed Hamiltonian (7)upto the 2nd order in $\beta$ using the BakerHausdroff Lemma as:

$$
\begin{aligned}
H^{\prime} & \approx H_{N C}+i \beta\left[\hat{O}, H_{N C}\right]-\frac{\beta^{2}}{2}\left[\hat{O},\left[\hat{O}, H_{N C}\right]\right] \\
& =\frac{\mathbf{p}^{2}}{2 M_{\theta \bar{\theta}}}+\frac{M_{\theta \bar{\theta}} \Omega_{\theta \theta}^{2} \mathbf{r}^{2}}{2}-S_{\theta \bar{\theta}} L_{z}-\lambda_{R}^{2} m
\end{aligned}
$$


where

$$
\begin{aligned}
& M_{\theta \bar{\theta}}=\left[\frac{1}{m}+m \omega_{0}^{2} \theta^{2}+\frac{2 \lambda_{R}^{2} m}{\left(\bar{\theta}-\frac{1}{\theta}\right)} \sigma_{z}\right]^{-1} \\
& \Omega_{\theta \bar{\theta}}^{2}=\left[\frac{1}{m}+m \omega_{0}^{2} \theta^{2}+\frac{2 \lambda_{R}^{2} m}{\left(\bar{\theta}-\frac{1}{\theta}\right)} \sigma_{z}\right]\left[\frac{\bar{\theta}^{2}}{m}+m \omega_{0}^{2}-\frac{2 \lambda_{R}^{2} m \frac{\bar{\theta}}{\theta}}{\left(\bar{\theta}-\frac{1}{\theta}\right)} \sigma_{z}\right] \\
& S_{\theta \bar{\theta}}=\left[\frac{\bar{\theta}}{m}+m \omega_{0}^{2} \theta+\lambda_{R}^{2} m \sigma_{z}\right] \\
& L_{z}=\left(x p_{y}-y p_{x}\right)
\end{aligned}
$$

It is noted that $\left[H^{\prime}, \sigma_{z}\right]=0$, and so we can use the spin on the $z$-direction to characterize the states. The Hamiltonian (9) takes the form

$$
H^{\prime}=\left(\begin{array}{cc}
H_{N C}^{\uparrow} & 0 \\
0 & H_{N C}^{\downarrow}
\end{array}\right)
$$

where

$$
H_{N C}^{\uparrow, \downarrow}=\frac{\mathbf{p}^{2}}{2}\left[\frac{1}{m}+m \omega_{0}^{2} \theta^{2} \pm \frac{2 \lambda_{R}^{2} m}{\left(\bar{\theta}-\frac{1}{\theta}\right)}\right]+\frac{\mathbf{r}^{2}}{2}\left[\frac{\bar{\theta}^{2}}{m}+m \omega_{0}^{2} \mp \frac{2 \lambda_{R}^{2} m \frac{\bar{\theta}}{\theta}}{\left(\bar{\theta}-\frac{1}{\theta}\right)}\right]-L_{z}\left[\frac{\bar{\theta}}{m}+m \omega_{0}^{2} \theta \pm \lambda_{R}^{2} m\right]-\lambda_{R}^{2} m
$$

The term $\lambda_{R}^{2} m$ is a constant for a particular system with a fixed spin orbit interaction. Then the Hamiltonians $H_{\theta \bar{\theta}}^{\uparrow, \downarrow}=H_{N C}^{\uparrow, \downarrow}+\lambda_{R}^{2} m$ can be solved exactly. It is possible to write any of the Hamiltonians $H_{\theta \bar{\theta}}^{\uparrow, \downarrow}$ as a sum of two separate one dimensional harmonic oscillators with frequencies $\Omega^{+}{ }_{\theta \bar{\theta}}$ and $\Omega^{-}{ }_{\theta \bar{\theta}}[20,21,22]$. As $H_{\theta \bar{\theta}}^{\uparrow}$ and $H_{\theta \bar{\theta}}^{\downarrow}$ are similar, we shall discuss only the case for $H_{\theta \bar{\theta}}^{\uparrow}$. For convenience, we are dropping the $\uparrow$ sign.

$$
H_{\theta \bar{\theta}}=\Omega_{\theta \bar{\theta}}^{+}\left[a_{\theta \bar{\theta}}^{\dagger} a_{\theta \bar{\theta}}+\frac{1}{2}\right]+\Omega_{\theta \bar{\theta}}^{-}\left[b_{\theta \bar{\theta}}^{\dagger} b_{\theta \bar{\theta}}+\frac{1}{2}\right]
$$

The explicit forms of the frequencies are given by

$$
\begin{aligned}
& \Omega_{\theta \bar{\theta}}^{+}=\sqrt{\Omega_{\theta \bar{\theta}}^{2}-S_{\theta \bar{\theta}}^{2}}+S_{\theta \bar{\theta}}, \\
& \Omega_{\theta \bar{\theta}}^{-}=\sqrt{\Omega_{\theta \bar{\theta}}^{2}-S_{\theta \bar{\theta}}^{2}}-S_{\theta \bar{\theta}}
\end{aligned}
$$

where $\Omega_{\theta \bar{\theta}}^{+} \neq \Omega_{\theta \bar{\theta}}^{-}$. Also, the anihilation operators are given by $a_{\theta \bar{\theta}}, b_{\theta \bar{\theta}}$ are given by

$$
\begin{aligned}
& a_{\theta \bar{\theta}}=\frac{1}{2 \sqrt{M_{\theta \bar{\theta}} \Omega_{\theta \bar{\theta}}^{+}}}\left[\left(p_{x}+i p_{y}\right)-i M_{\theta \bar{\theta}} \Omega_{\theta \bar{\theta}}^{+}(x+i y)\right] \\
& b_{\theta \bar{\theta}}=\frac{1}{2 \sqrt{M_{\theta \bar{\theta}} \Omega_{\theta \bar{\theta}}^{-}}}\left[\left(p_{x}-i p_{y}\right)-i M_{\theta \bar{\theta}} \Omega_{\theta \bar{\theta}}^{-}(x-i y)\right]
\end{aligned}
$$


The commutation relations between the creation operators $a_{\theta \bar{\theta}}^{\dagger}, b_{\theta \bar{\theta}}^{\dagger}$ and the annihilation operators $a_{\theta \bar{\theta}}, b_{\theta \bar{\theta}}$, are

$$
\left[a_{\theta \bar{\theta}}, a_{\theta \bar{\theta}}^{\dagger}\right]=\left[b_{\theta \bar{\theta}}, b_{\theta \bar{\theta}}^{\dagger}\right]=1
$$

with all other commutations being zero. The number operators $N_{\theta \bar{\theta}}^{+}=a_{\theta \bar{\theta}}^{\dagger} a_{\theta \bar{\theta}}$ and $N_{\theta \bar{\theta}}^{-}=$ $b_{\theta \bar{\theta}}^{\dagger} b_{\theta \bar{\theta}}$ satisfy the following eigenvalue eqation

$$
N_{\theta \bar{\theta}}^{ \pm}\left|n^{+}, n^{-}\right\rangle=n^{ \pm}\left|n^{+}, n^{-}\right\rangle
$$

with $n^{ \pm}=0,1,2 \ldots \ldots \ldots \ldots$

The energy eigenvalues of the Hamiltonian $H_{n c}$ given in eqn.(13) are

$$
E_{\theta \bar{\theta}}=\Omega_{\theta \bar{\theta}}^{+}\left(n^{+}+\frac{1}{2}\right)+\Omega_{\theta \bar{\theta}}^{-}\left(n^{-}+\frac{1}{2}\right)
$$

which is non-degenerate due to the presence of noncommutativity.

Now we can study some special cases concerning different relations between the two noncommutative parameters:

(i) If $\bar{\theta}=-m^{2} \omega_{0}^{2} \theta-\lambda_{R}^{2} m^{2}$, then $S_{\theta \bar{\theta}}=0$, and the energy eigenvalue is given by

$$
E_{\theta}=\left(n^{+}+n^{-}+1\right) \Omega_{\theta}
$$

where

$$
\Omega^{+}=\Omega^{-}=\Omega_{\theta}=\omega_{0} \sqrt{\left(1+m^{2} \omega_{0}^{2} \theta^{2}\right)^{2}+2 m^{2} \theta \lambda_{R}^{2}\left(m^{2} \omega_{0}^{2} \theta^{2}-1\right)}
$$

It can be seen from eqn.(22) when $\lambda_{R}=0$, the Hamiltonian is of an isotropic harmonic oscillator and our result coincides with that of ref.[23].

(ii)For, $\bar{\theta}=\frac{1}{\theta}+2 \lambda_{R} m \frac{1}{\sqrt{\theta}}, \Omega_{\theta \bar{\theta}}=S_{\theta \bar{\theta}}$, then

$$
E_{\theta}=\left(n^{+}-n^{-}\right) \Omega_{\theta}
$$

where

$$
\Omega^{+}=-\Omega^{-}=\Omega_{\theta}=\frac{1}{m \theta}+\frac{2 \lambda_{R}}{\sqrt{\theta}}+m \omega_{0}^{2} \theta+\lambda_{R}^{2} m
$$

The energy spectrum (23) is infinitely degenerate due to the constraint $\bar{\theta}=\frac{1}{\theta}+2 \lambda_{R} m \frac{1}{\sqrt{\theta}}$ which reduces to the constraint $\theta \bar{\theta}=1$ given in ref.[23] when $\lambda_{R}=0$.

(iii) The properties of the spectrum (12) depend on the ratio $\frac{\Omega_{\theta \bar{\theta}}^{+}}{\Omega_{\theta \bar{\theta}}^{-}}$. For irrational $\frac{\Omega_{\theta \bar{\theta}}^{+}}{\Omega_{\theta \bar{\theta}}^{-}}$the spectrum is non-degenerate while rational $\frac{\Omega_{\theta \bar{\theta}}^{+}}{\Omega_{\theta \bar{\theta}}^{-}}$leads to degeneracy [20]. Let us consider the latter case. We assume that $\frac{\Omega_{\theta \bar{\theta}}^{+}}{\Omega_{\theta \bar{\theta}}^{-}}=\frac{a^{-}}{a^{+}}$where $a^{-}, a^{+}$are relatively prime numbers. Let us put $\Omega_{\theta, \bar{\theta}}^{+}=a^{-} \sigma$ and $\Omega_{\theta, \bar{\theta}}^{-}=a^{+} \sigma$ where $\sigma$ is a constant. Then equation (12) gives

$$
E_{\theta, \bar{\theta}}=\sigma\left(a^{-} n^{+}+a^{+} n^{-}\right)+\sigma\left(\frac{a^{-}+a^{+}}{2}\right)
$$


The last term on the right hand side of equation (25) is an overall constant. So it follows that the spectrum (25) is degenerate, the degree of degeneracy being equal to the number of natural solutions $n^{ \pm}$to the equation $a^{-} n^{+}+a^{+} n^{-}=$constant.

\section{Quantum dot with spin-orbit interaction in a mag- netic field: Conditions for equivalence}

In the literature, an inverse relation between the noncommutative parameter and magnetic field has been obtained by comparing term by term of the Hamiltonian of the Landau problem and the Hamiltonian of an oscillator in noncommutative coordinate space. [24]. Moreover, considering the isotropic oscillator on a plane and taking both the position and momentum coordinates as noncommuative it was shown in [23] that there is a relation between the two noncommuative parameters with the magnetic field of the Landau problem, given by $\bar{\theta}=\frac{1}{\theta}=\frac{B}{2}$. This has motivated us to find whether there is a equivalence between the Hamiltonians representing a quantum dot with spin orbit interaction in noncommutative space with that of a quantum dot with spin orbit interaction in a magnetic field. In ref. [19] a partial diagonalization of the Hamiltonian representing a quantum dot with spin orbit interaction and Zeeman energy on an equal footing was derived analytically. Since we shall require the above mentioned results of ref. [19], we reproduce it here briefly.

The Hamiltonian representing a quantum dot with spin orbit interaction and Zeeman energy is given by,

$$
H_{B}=H_{0}+H_{R}+H_{z}
$$

where,

$$
\begin{aligned}
& H_{0}=\frac{1}{2 m}\left(P_{x}^{2}+P_{y}^{2}\right)+\frac{1}{2} m \omega_{0}^{2}\left(x^{2}+y^{2}\right) \\
& H_{R}=\lambda_{R}\left(P_{y} \sigma_{x}-P_{x} \sigma_{y}\right) \\
& H_{z}=\frac{\varepsilon_{z}}{2} \sigma_{z}
\end{aligned}
$$

Here, $\mathbf{P}=\mathbf{p}+\frac{e}{c} \mathbf{A}, \quad \mathbf{A}=\frac{B}{2}(-y, x, 0)$ This Hamiltonian can be diagonalized in spin space upto second order in the spin-orbit and the Zeeman parameters with the help of an unitary transformation[19]. It is to be mentioned that the energy scale corresponding to the spin-orbit and Zeeman interactions must be much smaller than that corresponding to the spin independent Hamiltonian $\left(H_{0}>>H_{R}, H_{z}\right)$, in order to neglect higher order terms introduced by the unitary transformation.

The transformed Hamiltonian is given by ,

$$
H_{B}^{\prime}=U^{\dagger} H_{B} U, U=e^{-i \beta \hat{O}}
$$

where,

$$
\hat{O}=\left(P_{x}-\frac{m \omega_{0}^{2}}{\varepsilon_{z}} y\right) \sigma_{x}+\left(P_{y}+\frac{m \omega_{0}^{2}}{\varepsilon_{z}} x\right) \sigma_{y}
$$




$$
\beta=-\frac{\lambda_{R} \varepsilon_{z}}{\omega_{0}^{2}+\omega_{c} \varepsilon_{z}-\varepsilon_{z}^{2}}, \quad \omega_{c}=\frac{e B}{m c}
$$

We expand the transformed Hamiltonian upto the 2nd order of $\beta$ using Baker Hausdrauff lemma as,

$$
\begin{aligned}
H_{B}^{\prime} & =H_{B}+i \beta\left[\hat{O}, H_{B}\right]-\frac{\beta^{2}}{2}\left[\hat{O},\left[\hat{O}, H_{B}\right]\right] \\
& =\frac{1}{2 M_{1}}\left(p_{x}^{2}+p_{y}^{2}\right)+\frac{1}{2} M_{1} \Omega_{1}^{2}\left(x^{2}+y^{2}\right)-S_{1}\left(x p_{y}-y p_{x}\right)+\frac{\varepsilon_{z}}{2} \sigma_{z}-K
\end{aligned}
$$

where,

$$
\begin{gathered}
\frac{1}{M_{1}}=\frac{1}{m}\left[1-\frac{2 \lambda_{R}^{2} m \varepsilon_{z}}{\omega_{0}^{2}+\omega_{c} \varepsilon_{z}-\varepsilon_{z}^{2}} \sigma_{z}\right] \\
\Omega_{1}^{2}=M_{1}^{-1}\left[m \omega_{0}^{2}+\frac{e^{2} B^{2}}{4 c^{2}}-\frac{\lambda_{R}^{2} \omega_{0}^{2} m}{\omega_{0}^{2}+\omega_{c} \varepsilon_{z}-\varepsilon_{z}^{2}} \frac{e B}{c} \sigma_{z}\right] \\
K=\lambda_{R}^{2} m\left[\frac{\omega_{0}^{2}+\omega_{c} \varepsilon_{z}}{\omega_{0}^{2}+\omega_{c} \varepsilon_{z}-\varepsilon_{z}^{2}}\right] \\
S_{1}=\frac{\lambda_{R}^{2} \omega_{0}^{2} m}{\omega_{0}^{2}+\omega_{c} \varepsilon_{z}-\varepsilon_{z}^{2}} \sigma_{z}-\frac{e B}{2 c M_{1}}
\end{gathered}
$$

Now we define,

$$
\begin{aligned}
H_{1 B}^{\prime} & =H_{B}^{\prime}+K-\frac{\varepsilon_{z}}{2} \sigma_{z} \\
& =\frac{1}{2 M_{1}}\left(p_{x}^{2}+p_{y}^{2}\right)+\frac{1}{2} M_{1} \Omega_{1}^{2}\left(x^{2}+y^{2}\right)-S_{1}\left(x p_{y}-y p_{x}\right) \\
& =\Omega_{1}^{+}\left(a_{1}^{\dagger} a_{1}+\frac{1}{2}\right)+\Omega_{1}^{-}\left(b_{1}^{\dagger} b_{1}+\frac{1}{2}\right)
\end{aligned}
$$

The Hamiltonian $H_{1 B}^{\prime}$ for the two spin eigenstates can be compared with the well known two dimensional harmonic oscillator problems. Here, the corresponding frequencies are given by

$$
\Omega_{1}^{+}=\sqrt{\Omega_{1}^{2}-S_{1}^{2}}+S_{1}, \quad \Omega_{1}^{-1}=\sqrt{\Omega_{1}^{2}-S_{1}^{2}}-S_{1}
$$

and the annihilation operators are given by

$$
\begin{aligned}
& a_{1}=\frac{1}{2 \sqrt{M_{1} \Omega_{1}^{+}}}\left[\left(p_{x}+i p_{y}\right)-i M_{1} \Omega_{1}^{+}(x+i y)\right], \\
& b_{1}=\frac{1}{2 \sqrt{M_{1} \Omega_{1}^{-}}}\left[\left(p_{x}-i p_{y}\right)-i M_{1} \Omega_{1}^{-}(x-i y)\right]
\end{aligned}
$$

Correspondingly we can define the creation operators also and they satisfy the usual commutation relations. 
Eqn.(36) can be written as

$$
\begin{aligned}
H_{1 B}^{\prime} & =\frac{p^{2}}{2 M_{1}}+\frac{M_{1} \Omega_{1}^{2} r^{2}}{2}-S_{1} L_{z} \\
& =\Omega^{+}\left(a_{1}^{\dagger} a_{1}+\frac{1}{2}\right)+\Omega^{-}\left(b_{1}^{\dagger} b_{1}+\frac{1}{2}\right)
\end{aligned}
$$

The energy eigenvalues of this Hamiltonian $H_{1 B}^{\prime}$ is given by

$$
E_{1 B}^{\prime}=\Omega_{1}^{+}\left(n^{+}+\frac{1}{2}\right)+\Omega_{1}^{-}\left(n^{-}+\frac{1}{2}\right)
$$

where $n^{ \pm}=0,1,2, \ldots$

The similarity of the energy spectrum corresponding to the Hamiltonians (9) and (26) can be exploited to find a relation between the noncommutative parameters and spin orbit coupling strength, and the magnetic field strength. Comparing the like terms of the Hamiltonians (9) and (26) we get

$$
\begin{gathered}
\frac{2 \lambda_{R}^{2} \varepsilon_{z}}{\omega_{0}^{2}+\omega_{c} \varepsilon_{z}-\varepsilon_{z}^{2}}+m \omega_{0}^{2} \theta^{2}+\frac{2 m \lambda_{R}^{2}}{\bar{\theta}-\frac{1}{\theta}}=0 \\
\frac{e^{2} B^{2}}{4 m}\left(1-\frac{2 \lambda_{R}^{2} m \varepsilon_{z}}{\omega_{0}^{2}+\omega_{c} \varepsilon_{z}-\varepsilon_{z}^{2}}\right)+m \omega_{0}^{2}-\frac{e B m \omega_{0}^{2} \lambda_{R}^{2}}{\omega_{0}^{2}+\omega_{c} \varepsilon_{z}-\varepsilon_{z}^{2}}=\bar{\theta}^{2}+m \omega_{0}^{2}-\frac{2 m \lambda_{R}^{2} \overline{\bar{\theta}}}{\bar{\theta}-\frac{1}{\theta}} \\
\frac{m \omega_{0}^{2} \lambda_{R}^{2}}{\omega_{0}^{2}+\omega_{c} \varepsilon_{z}-\varepsilon_{z}^{2}}-\frac{e B}{2 m}\left(1-\frac{2 m \lambda_{R}^{2} \varepsilon_{z}}{\omega_{0}^{2}+\omega_{c} \varepsilon_{z}-\varepsilon_{z}^{2}}\right)=\left(\frac{\bar{\theta}}{m}+m \omega_{0}^{2}+\lambda_{R}^{2} m\right) \\
\omega_{0}^{2}+\omega_{c} \epsilon_{z}-\epsilon_{z}^{2}=2 \lambda_{R}^{2} m \epsilon_{z}
\end{gathered}
$$

The eqns.(37),(38), (39) and (40) are consistent if the folowing equation is satisfied

$$
e B\left(\bar{\theta}+m^{2} \omega_{0}^{2} \theta+\lambda_{R}^{2} m^{2}\right)+\bar{\theta}^{2}+\frac{\bar{\theta}}{\theta}\left(1+m^{2} \omega_{0}^{2} \theta^{2}\right)=0
$$

It is to be noted that if $\bar{\theta}=0$, then the equivalence condition (44) reduces to

$$
\lambda_{R}^{2}=-\omega_{0}^{2} \theta
$$

which is independent of $B$. This result is consistent with that obtained in ref. [21] which indicates that the origin of momentum noncommutativity is due to the magnetic field $[21]$. 


\section{Conclusion}

In this article, a quantum dot,in harmonic confinement, with Rashba spin -orbit interaction on a noncommutative phase space is considered which has not been reported in the literature so far our knowledge goes. We have obtained the energy spectrum which is nondegenerate. But the spectrum becomes degenerate when the noncommutative parameters $\theta$ and $\bar{\theta}$ satisfy certain constraints. Also we have compared our system with a quantum dot with Rashba spin-orbit interaction in a magnetic field and derived the condition for equivalence of the two systems when the spin -orbit interaction strength $\lambda_{R}$ is small. We intend to analyse the energy spectrum of a quantum dot in anisotropic confinemnet on a noncomutative plane in future.

\section{Acknowledgements}

B.K.Pal acknowledges Council of Scientific and Industrial Research(CSIR), India for financial support.

\section{References}

1. M.R.Douglas and N.A.Nekrasov, Rev.Mod.Phys. 73977 (2001)

R. Szabo Phys. Rep. 378207 (2003)

A.P.Balachandran, T.R.Govindarajan, C.Molina and P.Teotonio-Sobrinho, JHEP 0410072 (2004)

2. E.Witten, Nucl.Phys. B460 33 (1996)

M.R.Douglas and C.M.Hull, JHEP 02008 (1998)

C.Chu and P.Ho, Nucl.Phys. B550 151 (1999); ibid B568 447 (2000)

N.Seiberg and E.Witten, JHEP 09032 (1999)

3. I.Antoniads, N.Arkani-Hamed, S.Dimopoulas and G.R.Dvali, Phys.Letts. B436 257 (1998)

4. A.Connes, M.R.Douglas and A.Schwarz, JHEP 02003 (1998)

5. B. Chakrabarty, S. Gangopadhyay and A. saha, Phys. Rev. D 70, 107707 (2004)

O.F. Doyi and A. Jellal J. Math Phys. 434592 (2004) 
6. H.S.Snyder, Phys.Rev. D71 38 (1946)

C.N.Yang, Phys.Rev. D72 874 (1947)

7. F. Delduc et. al. J.Phys. Conf. Series 103012020 (2008)

8. The literature is very extensive, some papers are:

G.V.Dunne,J.Jackiw and C.Trugenberger, Phys.Rev.D41 661 (1990).

H.Falomir,J.Gamboa,M.Loewe,F.Mendez, Phys.Rev.D66 045018 (2002)

F.S.Bemfica,H.O.Girotti, J.Phys.A38 L539 (2005).

C.Duval And P.A.Horvathy, Phys.Lett.B547 306 (2002)

C.Duval And P.A.Horvathy, J.Phys.A34 10097 (2001),ibid,Phys.Lett. C.Duval And

P.A.Horvathy,Phys.Lett.B479 284 (2000)

D.V.Vassilevich,JHEP 0805093 (2008)

F.G.Scholtz, B.Chakraborty, S.Gangopadhyay and A.Hazra, Phys.Rev.D71 085005 (2005)

F.G.Scholtz, L.Gouba, A.Hafver and C.M.Rohwer, J.Phys.A:Math.Theor,42 175303(2009)

P.A.Horvathy and M.S.Plyushchay,Nucl.Phys B714 269(2005)

B.Basu,S.Ghosh and S.Dhar,Europhys.Lett.76 395 (2006)

S.Dhar,B.Basu and S.Ghosh ,Phys.Lett.A 371406 (2007)

9. L. Lucjan, P. Hawrylak, A. Wojs, Quantum Dots, Springer-Verlag, Berlin Heidelberg (1998)

T.Chakraborty, Quantum Dots A survey of the properties of artificial atoms, Elsevier, The Netherlands (1999).

10. G.Dresselhaus, Phys.Rev. 100 (1955) 580

11. E.I.Rashba, Fiz.Tverd.Tela(Leningrad) 21224 (1960), Sov.Phys.Solid State 21109 (1960); Yu.A.Bychkov and E.I.Rashba, J.Phys.C 176039 (1984)

12. A.V.Chaplik and L.I.Magarill, Phys.Rev.Letts. 96126402 (2006)

13. T. Chakraborty and P. Pietilainen, Phys. Rev.B 71113305 (2005)

14. B. Basu and B. Roy, Eur.J. Phys. (accepted) (2009)

15. Jian-zu Zhang, Phys. Lett. B 584 204(2004) 
16. J. Gomis and T.Mehen, Nucl. Phys. B 591265 (2000), M. Chaichian.et.al. Eur. Phys. J.C 20767 (2001)

17. T. Curtright et.al. Phys. Rev. D 58025002 (1998)

18. I.L. Aleiner and V.I. Fal'ko, Phys. Rev. Let. 87256801 (2001)

19. M. Valin-Rodriguez, Phys.Rev.B70 033306 (2004).

20. A. Kijanka and P. Kosinski, Phys. Rev. D 70, 1277702 (2004)

21. V.P. Nair and A.P. Polychronakos, Phys. Lett. B505 267 (2001)

22. A. Smailagic and E. Spallucci, Phys. Rev. D 65, 107701 (2002)

23. P.Giri and P. Roy Euro.Phys. J. C 57835 (2008)

24. J.Gamboa et. al. Mod. Phys. Lett. A 162075 (2001) 